\title{
Performance analysis of TCP with delayed acknowledgments in multi-hop ad-hoc networks.
}

\begin{abstract}
The acknowledgment strategy has great potential to increase TCP throughput when it runs over 802.11 MAC protocol. In particular, TCP acknowledgments (ACK) carry out an extensive number of medium accesses as they compete in the same route as data packets for media. In this paper, we first propose a dynamic TCP-MAC interaction strategy which tries to reduce the number of induced ACKs by monitoring the channel condition. To this end, the total collision probability collected along the path from sender to receiver in MAC layer has been used to properly set the number of delayed ACKs (DA) in TCP. Based on the estimated collision probability, TCP sender dynamically adjusts itself to the channel condition by delaying less ACKs in high traffic conditions and more ACKs in low traffic conditions. The simulation results show a throughput improvement up to $15 \%$ over the existing method called Dynamic Adaptive Acknowledgment (TCP-DAA) and much more over the regular TCP in different scenarios dealing with a dynamic loss rate. In addition, we show that our proposed strategy does not always benefit from a fixed delay policy along with a fixed congestion window size. In fact, the optimal number of delayed ACKs is based on the path length of a TCP connection and a large delay window may solely improve TCP throughput in short ranges with less number of flows. However, in a longer path congestion window limit provides more throughput gain.
\end{abstract}

Keyword: Multi-hop ad-hoc networks; Transport Control Protocol (TCP); Delayed Acknowledgment (DA); 802.11 MAC protocol. 